\title{
La huída del Mordor caribeño: el exilio y la diáspora dominicana en The Brief Wondrous Life of Oscar Wao, de Junot Díaz
}

\author{
Carmen M. MÉndeZ GARCÍA \\ Departamento de Filología Inglesa II \\ Universidad Complutense de Madrid \\ cmmendez@filol.ucm.es
}

\section{RESUMEN}

Desde el comienzo de su historia como colonia británica, Estados Unidos ha recibido un gran número de exiliados que, en numerosas ocasiones, han empleado la literatura para narrar su experiencia de diáspora original y la posterior búsqueda de raíces de los descendientes de aquellos que en su día iniciaron el proceso de migración. Analizaremos la obra The Brief Wondrous Life of Oscar Wao (2007), del escritor dominicano-americano Junot Díaz, como un terreno de tensión entre el país que fuerza el exilio (la República Dominicana) y el de acogida (Estados Unidos), algo reflejado también en el complejo equilibrio de la identidad de los protagonistas, escindidos entre dos culturas. Estudiaremos, asimismo, cómo el texto principal y las numerosas y larguísimas notas a pie de éste, algo no habitual en la novela como género, representan la tensión entre voz autorial y otras voces relegadas a los márgenes, reflejando la tensión entre dictador y disidentes exiliados o desaparecidos de la República Dominicana durante el Trujillato.

Palabras clave: Junot Díaz, exilio, literatura dominicano-americana, el Trujillato en la literatura.

Escape from Caribbean Mordor: Exile and the Dominican Diaspora in Junot Diaz's The Brief Wondrous Life of Oscar Wao

\begin{abstract}
Since the beginning of their history as British colonies, the United States have taken in a large number of exiles, who have often used fiction to recount their diasporic experience and the subsequent search for roots by the descendants of those who migrated. We will analyze The Brief Wondrous Life of Oscar Wao (2007), by Dominican-American writer Junot Díaz, as a locus of tension between the country that forces into exile (the Dominican Republic) and


the country who takes in (the United States), also reflected in the complex identity of the protagonists, split between two cultures. We will examine, as well, the connection between the main text and the abundant and long footnotes -something quite unusual in the novel as a genre-, and expound how this reflects the tension between the authorial voice and other voices which are banished into the margins, mirroring the tension between the dictator and exiled or disappeared dissidents in the Dominican Republic during the Trujillato.

Key words: Junot Díaz, exile, Dominican-American Literature, Trujillato in literature.

[Trujillo] was our Sauron, our Arawn, our Darkseid, our Once and Future Dictator, a personaje so outlandish, so perverse, so dreadful that not even a sci-fi writer could have made his ass up. (Díaz 2007: 2)

Resulta complicado, a estas alturas, escribir nada sobre los Estados Unidos no sólo como país de inmigración, sino también como país de acogida a exiliados, sin caer en la obviedad o en análisis ya demasiado trillados. Igualmente evidente es que el exilio ha tendido a centrarse geográficamente en territorio norteamericano alrededor de tres grandes zonas que muestran, claramente, una identidad propia como resultado de dicho exilio: la costa Este, especialmente el territorio más cercano a la ciudad de Nueva York; la zona de Florida, más concretamente Miami; y California, demasiado amplia territorialmente para buscar un solo foco, pero con mayor protagonismo de las áreas urbanas de San Francisco y Los Angeles. Estados Unidos no sólo no es una excepción al flujo migratorio, tan característico de los siglos XX y $\mathrm{XXI}$, sino que fundamenta su historia, primero como colonia inglesa, y posteriormente como nación, en la llegada de inmigrantes, de exiliados, de poblaciones desplazadas, y de grupos migratorios y diaspóricos. Resulta, por tanto, de sumo interés explorar cómo distintas comunidades o autores hayan podido enfocar "su" historia de exilio y diáspora expresándola a través de la literatura, una expresión que siempre aúna, necesariamente y por más que el autor se centre en su propia experiencia, lo grupal y lo individual. George O'Brien ha definido el exilio como "a movement of the mind, a cultural reaction, a metonym for the restlessness, disaffection, isolation and self-respect" (O’Brien 1988: 99). Es en este sentido metonímico como analizaremos la obra The Brief Wondrous Life of Oscar Wao (2007), del escritor dominicano-americano Junot Díaz, estudiando cómo en el texto se mezclan el exilio (real, geográfico, de Santo Domingo a New Jersey) de una generación con el exilio (cultural, de aislamiento, "a movement of the mind", siguiendo a O'Brien) de otra.

La costa este de los Estados Unidos, y específicamente la ciudad de Nueva York, ha asistido no sólo a la llegada masiva de inmigrantes dado su carácter mítico de puerta de entrada a los Estados Unidos (simbolizado por la Estatua de la Libertad y el centro de inmigración de Ellis Island), sino que también, quizá precisamente por su relevancia como centro económico y por el auténtico crisol que se configura en su espacio, ha resultado foco de interés para grupos que provenían del exilio y movimientos diaspóricos. En las siguientes páginas, sin embargo, no nos referiremos al 
estado ni a la ciudad de Nueva York (donde los dominicanos constituyen el segundo grupo más importante de latinos, por detrás sólo de los portorriqueños), sino a la muy cercana New Jersey, un estado limítrofe donde se han ubicado gran parte de las comunidades inmigratorias o diaspóricas que han llegado a Estados Unidos tras pasar por el portal que es Nueva York, siguiendo la admonición del famoso soneto de Emma Lazarus "The New Colossus", publicado en 1883 e inscrito en el pedestal de la Estatua de la Libertad en 1903: "Give me your tired, your poor / Your huddled masses yearning to breathe free / The wretched refuse of your teeming shore".

Nada mejor para hablar de movimientos diaspóricos en New Jersey que esta novela de Junot Díaz publicada en 2007. Agotaría el espacio destinado a este artículo listar la gran cantidad de premios que ha obtenido una obra tan reciente, y sin embargo ya tan conocida: aparte del afamado premio Pulitzer, se podrían destacar también el National Book Critics Circle Award a la mejor novela de 2007, el Massachusetts Book Prize for Best Fiction de 2008, y la elección de la novela por parte de las prestigiosas revistas Time y New York Magazine como mejor novela de 2007. Desde un punto de vista académico, en la conferencia nacional de la MLA (Modern Language Association, una de las asociaciones de literatura más importantes de EE.UU., y auténtico referente académico e investigador de este país) de 2008, en San Francisco, The Brief Wondrous Life of Oscar Wao había recibido la suficiente atención crítica, tan solo un año después de su publicación, como para merecer un panel exclusivo, situación no muy habitual en autores sin una larga trayectoria, algo que no es el caso de su autor. En Time, el crítico Lev Grossman definió la novela como:

so astoundingly great that in a fall crowded with heavyweights -Richard Russo, Philip Roth- Díaz is a good bet to run away with the field. You could call [it] the saga of an immigrant family, but that wouldn't really be fair. It's an immigrantfamily saga for people who don't read immigrant-family sagas. (Grossman 2007)

Las historias de la primera colección de cuentos de Díaz, Drown, publicada más de una década antes, en 1996, ficcionalizan la infancia y adolescencia del autor, también, como el protagonista de los relatos, pobre y abandonado por su padre, mientras trata de adaptarse a una nueva vida en New Jersey: resulta notable que todos los personajes de la obra vivan en el campo o en barrios marginales, tanto en la República Dominicana como en Nueva York, en espacios que podrían definirse como abyectos, como una especie, en el caso de aquellos viviendo ya en EE.UU., de exilio dentro del exilio. El texto fue traducido al español como Los Boys (Barcelona: Mondadori), y mientras que sirvió para llamar la atención de numerosos lectores hacia el autor, recibió críticas de muy diversa índole. The Brief Wondrous Life of Oscar Wao, sin embargo, se ha convertido en un unánime fenómeno editorial y crítico, ha entrado en el currículum de las universidades en los cursos que estudian literatura contemporánea (sea o no escrita por minorías), y sigue resultando atractivo para el gran público, sobre todo el más joven, por sus juegos intertextuales (como luego veremos) que incluyen guiños a la cultura popular, con menciones constantes a obras de fantasía, cómics y ciencia ficción. $Y$ todo esto en una novela que dedica, al menos, un tercio de su duración a hablar no de la vida breve pero llena de maravillas de Oscar Wao 
mientras crece en New Jersey, sino de la vida, llena de sufrimiento y represión, de su familia en Santo Domingo, y de sus problemas con las esferas de poder de la isla. Asistimos así a la historia del declive profesional y personal de Abelard, el abuelo de Oscar, que es arrestado (y, posteriormente, encarcelado y torturado hasta la muerte) por motivos políticos, por "the Bad Thing he said about Trujillo" (Díaz 2007: 212), es decir, por bromear en público acerca de la desaparición inexplicable de enemigos del régimen. Pero también el destino de Abelard se debe a motivos carnales (intentar evitar que el dictador disponga a su antojo de Jacqueline Cabral, su hija y hermana de Beli, de la que se ha encaprichado) que enfatizan el poder absoluto sobre los cuerpos y las almas del dictador y su círculo en la isla. Completando este episodio de los antepasados de Oscar en la República Dominicana con la narración del posterior exilio de su madre a los Estados Unidos, Díaz relaciona dos mundos bien diferenciados, pero en el fondo íntimamente unidos: la tierra de origen, la República Dominicana, plagada de fantasmas, que se muestran una y otra vez en las pesadillas de los protagonistas que viven ya en el exilio; y la tierra de libertad, sueños y esperanza simbolizada por New Jersey, donde han acabado los personajes como parte de lo que se ha dado en llamar la gran Diáspora dominicana.

La obra, así pues, refleja las vivencias de una familia de dominicano-americanos (el cuarto grupo hispano en número de residentes en Estados Unidos) en la ciudad de Paterson, en New Jersey, que hiciera conocida para las letras norteamericanas el poeta William Carlos Williams entre 1946 y 1958. Paterson es, de hecho, la octava ciudad de Estados Unidos con mayor porcentaje de dominicano-americanos: resulta lógico, por otro lado, desde un punto de vista geográfico, que todas las ciudades que integran esta particular clasificación estén ubicadas en la costa Este, bien en Nueva York (Sleepy Hollow, Haverstraw, el Bronx o Manhattan) o New Jersey (Perth Amboy, Union City, o el mismo Paterson), con la única excepción de la muy cercana Lawrence, en el cercano estado de Massachusetts (Shorter 2005).

La acción de la novela tiene lugar, de forma alterna, en el presente en el ya mencionado Paterson, y en Santo Domingo tanto en el presente como en el pasado, durante la época del dictador Trujillo (1930-1961), reflejando en los dos últimos escenarios la herencia de la represión, mediante torturas, amenazas y coacciones brutales, de éste en el actual gobierno y cultura de la isla, mediante una reflexión sobre la megalomanía de Trujillo. En la vida real, según se nos informa en la primera nota a pie de la novela, esta megalomanía se tradujo, entre otras cosas, en "renombrar" con su apellido o el de algunos de sus familiares numerosas zonas del país, como Santo Domingo, que pasó a llamarse temporalmente "Ciudad Trujillo", o Pico Duarte, el pico más alto del Caribe, que fue renombrado como "Pico Trujillo". También dedica la novela un amplio espacio de reflexión a los métodos de control de los disidentes que incluían, a menudo, la tortura y la casi imposible elección entre la muerte o el exilio.

1 "Famous for changing ALL THE NAMES of ALL THE LANDMARKS in the Dominican Republic to honor himself $[\ldots]$ for making ill monopolies out of every slice of the national patrimony [...] for expecting, no, insisting on absolute veneration from his pueblo" (Díaz 2007: 2). 
The Brief Wondrous Life of Oscar Wao no es la única, ni seguramente todavía la más conocida representación literaria del llamado Trujillato, aunque como parte de una posible intención didáctica, Junot Díaz dirige gran parte de las notas a pie, parte integral de la narrativa, a aquellos "who missed our mandatory two seconds of Dominican history" (Díaz 2007: 2). Posiblemente, La fiesta del chivo (2000), del reciente premio Nobel Mario Vargas Llosa, quedara en primer lugar en una hipotética clasificación, o incluso sería más conocida la obra de otra dominicano-americana, Julia Álvarez, con su In the Time of Butterflies (1994), sobre el asesinato de las "mariposas", las conocidas hermanas Mirabal. Álvarez y su familia huyeron a los Estados Unidos escapando del Trujillato, y su obra de 1994 supone la búsqueda de respuestas y una posible "reconciliación" con las personas oprimidas por el régimen, aunque al contrario que los Mirabal, la familia de Álvarez encontrara una solución en el exilio ${ }^{2}$. La dictadura de Rafael Leónidas Trujillo ha sido definida como "one of the most hermetically tyrannical states in the history of Latin America" (Lifshey 2008: 435), y es el tema fundamental de otras obras como El Masacre se pasa a pie (1973) de Freddy Prestol Castillo, el relato "La Mancha Indeleble" (1980) del también dominicano Juan Bosch (exiliado durante parte de la dictadura de Trujillo en Puerto Rico), The Farming of Bones (1998) de la haitianaamericana Edwige Danticat (sobre el genocidio de haitianos en la República Dominicana conocido como "el corte"), o incluso Galíndez (1991) del escritor español Manuel Vázquez Montalbán. Gran parte de estas obras se podrían englobar en el género, bien establecido en literatura hispanoamericana, de la "novela de dictador" (Lifshey 2008: 438), y es en parte dentro de esta tradición donde se engloba la novela de Díaz, que sin embargo también se inscribe como antes vimos dentro del género de la saga familiar, que goza de excelente salud en la literatura hispanoamericana, con obras que se extienden desde Cien años de soledad (1967) de Gabriel García Márquez, hasta La Casa de los espíritus (1982) de Isabel Allende, por ejemplo. La historia del exilio dominicano se vincula en la obra de Díaz, por tanto, a través del uso de géneros bien establecidos en la literatura hispanoamericana como las novelas de dictador y las saga familiares, con la historia de los De León, protagonistas de la obra, en un resultado que también bebe del realismo mágico, y que es así mismo un bildungsroman; todo ello para crear un texto que refleja la imposibilidad por parte del exiliado renunciar a su "unextinguishible longing for elsewheres" (Díaz 2007: 77).

Tanto Junot Díaz como Julia Álvarez nacieron en Santo Domingo y fueron llevados a Estados Unidos durante su infancia. El escritor cubano-americano Gustavo Pérez-Firmat ha elegido para referirse a este tipo de exiliados la etiqueta "generación uno y medio", término que destaca no sólo la dificultad de tener que negociar la transición de la infancia a la edad adulta, sino de hacerlo pasando de un entorno socio-cultural a otro (Pérez-Firmat 1994: 4). Para Pérez-Firmat, la negociación de culturas específica de esta generación "uno y medio" es lo que convierte a sus inte-

2 Julia Álvarez retoma la huída y el exilio de otra familia dominicana, los García, en How the García Girls Lost their Accents (1991). 
grantes en miembros de una "cultura con guión" (Pérez Firmat 1994: 13): no son ni uno, ni otro, lo que paradójicamente facilita la "negociación" entre ambas culturas; para otros críticos, sin embargo, mientras que los adultos son capaces de traer el pasado con ellos al llegar a una nueva cultura,

there is no diaspora of children. Their individual survival comes at the cost of their old identity [...]. They receive the priceless gift of life [and] a new future opens up where only bleakness or extinction may have threatened before, but only by means of a substitution they did not choose [...]. They live on in the identity accepted [...] from a new culture, aware that something will always persist in them of a destiny broken off by fate, always irreparably lost though never completely overcome. (Bullock apud Fehervary 2008: 15)

Quizá sea éste el motivo por el que escritores como Díaz o Álvarez se ven obligados a re-imaginar el pasado (directo de su familia, en el caso de Díaz; o histórico de su nación, en el caso de Álvarez) a través de sus obras. Habitualmente las obras escritas por esta generación enfatizan la dificultad, frente a la generación anterior (preocupada por integrarse en la sociedad del nuevo país), de habitar el espacio liminal en el que se ven forzados a existir, que no es ni el país de origen (en este caso, de exilio) de sus antepasados, ni los EE.UU. donde, por ubicarse dentro de una comunidad de expatriados, siguen existiendo de alguna manera en los márgenes de la sociedad ${ }^{3}$. $\mathrm{Al}$ mismo tiempo, estos autores han de fundamentar su conocimiento y descripción del colectivo dominicano-americano desde posiciones de exilio, no de migración convencional (Ink 2004: 782). Estos escritores "regresan al pasado, no para confrontar la historia o lamentar la pérdida como lo habían hecho los escritores del grupo anterior, sino para encontrar una oportunidad de balancear [sic.] las dos culturas que viven simultáneamente en ellos" (Álvarez Borland 2003: 42). Así lo aplica Díaz a su propia experiencia:

I have a sense of the Dominican [...] it's not much of a theory, more a collection of words, a dot dot dash code that I use to, in another way, decipher a larger code, which is the Dominican experience, the Dominican diasporic experience, and the American experience, all hooked together. (Lewis 2002)

The Brief Wondrous Life of Oscar Wao supuso un inesperado éxito editorial para Díaz, que se convirtió en el primer dominicano-americano en ganar el premio Pulitzer. En 2008, transformado ya en figura mediática, Díaz reflexiona sobre su éxito como escritor en términos no alejados del "sueño americano" al que se exponen

3 Al hablar de Julia Álvarez, Suarez destaca también cómo parte del problema de los jóvenes dominicano-americanos puede ser que la experiencia de exilio se complica por el convencimiento de que la huída por motivos políticos es, en cierto modo, y una vez que se ha llegado a tierra salva, un tipo de inmigración "privilegiada", frente al estigma que supone la inmigración por motivos meramente económicos (Suarez 2004: 126). Es además más sencillo para los dominicanos que para los emigrantes de otros países de Hispanoamérica obtener la ciudadanía norteamericana sin tener que renunciar a su nacionalidad. 
comunidades inmigrantes y diaspóricas, definiéndose como inmigrante a los Estados Unidos en los siguientes términos:

I can safely say I've seen the US from the bottom up [...] I may be a success story as an individual. But if you adjust the knob and just take it back one setting to the family unit, I would say my family tells a much more complicated story. It tells the story of two kids in prison. It tells the story of enormous poverty, of tremendous difficulty. (Ying 2010)

Junot Díaz no oculta, en su mismo título, las referencias temáticas a "The Short and Happy Life of Francis Macomber", de Ernest Hemingway. En este relato, publicado por primera vez en 1936, el protagonista, el Francis Macomber del título, tan sólo encuentra el verdadero sentido de una vida enraizada en la cobardía cuando se enfrenta a la adversidad, por primera vez, desde una pose heroica que, in extremis, le costará la vida pero que al mismo tiempo dotará de un cierto significado trágico a su muerte. Algo similar sucede al protagonista, Oscar de León, que hallará el sentido de su vida en un último e inesperado acto heroico que, paradójicamente, replica experiencias de su familia en su isla de origen, y precisamente será tan solo en los momentos previos a su muerte cuando sea capaz de hablar perfectamente, sin acento y de forma articulada, el idioma de sus ancestros: "[t] he words coming out like they belonged to someone else, his Spanish good for once" (Díaz 2007: 321). "Wao", el apodo del protagonista, Oscar De León, es, también, una deformación de "Wilde", por el parecido que sus compañeros ven con otro Oscar, el conocido autor irlandés. Con estas referencias literarias, Díaz (profesor de literatura creativa en el MIT) mezcla, en la vena del espíritu postmoderno, lo que se ha dado en llamar "alta" literatura (como el extracto de "The Schooner Flight" (1980), poema del reconocido autor caribeño Derek Walcott que se encuentra al comienzo de la novela) con la denominada "literatura popular" (ya en la cita del cómic Los Cuatro Fantásticos con la que se inicia el libro: "Of what import are brief, nameless lives... to Galactus??"). Así, Díaz se ubica dentro de una tradición claramente angloamericana, canónica pero también anclada en lo popular, al tiempo que introduce elementos (y una gran parte de la trama) decididamente dominicanos, como el uso del castellano y de palabras y conceptos que parten del Caribe, y que acaban conformando una obra mixta, que habla de exilios, políticos, pero también lingüísticos o exilios interiores, como es el caso del joven protagonista.

Tras una primera sección donde el narrador Yunior de las Casas (novio ocasional de la hermana de Oscar, Lola, y amigo y "protector" del propio Oscar) comienza a contar la breve historia del protagonista, asistimos en las páginas intermedias del libro a las vivencias, narradas en primera persona, de Lola durante una larga visita a Santo Domingo, donde vive con una prima de su abuelo, La Inca. De esta manera, el libro intercala vivencias actuales de la generación más joven de los De León, Oscar y Lola, y de su madre (Hypatia Belicia Cabral, "Beli") en New Jersey con las circunstancias (narradas en pasado) que motivaron el exilio de la familia de Santo Domingo, con protagonismo absoluto en el segundo caso de los abusos del dictador Trujillo, que asesinó a la familia de Beli, forzándola a criarse con una serie 
de familias de acogida hasta ser rescatada por una prima de su padre (La Inca), y que posteriormente hace que, debido a la relación ilícita entre Beli y uno de los cuñados de Trujillo, ésta tenga que abandonar la isla. El libro comienza a cerrar el círculo en la tercera y última sección, con el regreso y última visita de Oscar a Santo Domingo; regreso que, por otra parte, terminará por costarle la vida en circunstancias muy parecidas a las que hicieron que su madre tuviera que exiliarse a New Jersey, precisamente en el mismo lugar donde aquélla recibió una brutal paliza, y donde Oscar ya había sido llevado en una ocasión sin que demostrara la valentía que muestra en los últimos momentos de su segunda visita. La repetición del contexto geográfico, de las motivaciones (amorosas) que provocan la suerte de ambos protagonistas, e incluso la aparición fantasmagórica, en la tradición del realismo mágico, de una mangosta en episodios similares, aporta una estructura circular de exilio y regreso, que por otra parte ya habían iniciado otros personajes del libro como Lola. El texto se cierra recurriendo a un capítulo de tintes tradicionales (que contrasta con la inclusión ya mencionada de elementos de la cultura popular, y que por virtud de dicha mezcla ubican el libro dentro de lo que se ha dado en llamar lo postmoderno), en el que se informa al lector del final de cada uno de los personajes, culminando, en su última frase, con otra referencia inescapable para cualquiera siquiera mínimamente versado en la tradición literaria angloamericana: "The beauty! The beauty!" (Díaz 2007: 335), un eco del final de Heart of Darkness (1902) de Joseph Conrad, una obra, también, sobre dictadores, exilios y viajes de descubrimiento personal a través del encuentro con la oscuridad que subyace en el ser humano.

The Brief Wondrous Life of Oscar Wao utiliza de forma insistente, como ya hemos adelantado (y quizá en esto radique parte de su éxito), una plétora de referencias a la cultura popular, a los cómics de superhéroes, a la literatura fantástica, a la ciencia ficción, al manga, a los juegos de rol, a la literatura llamada "de género" o "de fórmula" (novela de horror, o novela romántica), a los juegos de mesa y a películas de culto como The Matrix (1999). Muchos lectores, inicialmente atraídos por el éxito del libro, han reconocido sentirse superados por la cantidad de referencias ${ }^{4}$, por el número de géneros utilizados, la alternancia de narradores, el abusivo número de notas a pie, larguísimas y digresivas (si bien excepcionalmente divertidas e iluminadoras) y por la ausencia de una narrativa principal que "guíe" al lector por el relato $^{5}$. A las objeciones acerca de esta falta de coherencia narrativa contesta Junot Díaz:

The idea of a master narrative scares the hell out of me. As a child of the dictatorship of course it's going to scare the hell out of you. And so [you have] the foot-

\footnotetext{
4 Resulta imposible analizar completamente el complejo sistema de referencias e intertextualidad que encontramos en The Brief Wondrous Life of Oscar Wao, hasta tal punto que se ha creado ya una versión anotada del texto, disponible en internet, que pese a estar en constante desarrollo no agota, sin embargo, todas las referencias de la obra: http://www.annotated-oscar-wao.com/.

5 Kakutani ha definido el libro como una novela donde "Mario Vargas Llosa meets Star Trek meets David Foster Wallace” (Kakutani 2007).
} 
notes competing with the narrative of the master overtext; [and] the book [countering] its own authoritativeness by directing you to other books on the same theme, competing books. (Díaz 2008)

Para Díaz, esta huida del narrador "dictatorial" es una de las lecciones "that we learned in this kind of post-dictatorship / dictatorship-traumatised country" (Díaz 2008). Díaz insiste en atacar esta noción de la historia en la que el texto "is sacrosanct and one. That was the national myth of the Dominican Republic as long as this story's going on, this story is sacrosanct and the only one. Competing narratives were dismissed, marginalized -they were assassinated" (Díaz 2008).

Las referencias intertextuales no son, pues, intención por parte del autor solo de descubrir al lector el tipo de obras que forman parte de su educación cultural, sino que también aspiran a crear "metaphors and lenses through which to interpret the world" (Díaz 2008), y también surgen del deseo de cambiar la forma de leer: la digresión, las notas a pie, funcionan como una manera de "sacar" al lector de la voz dictatorial que impone un narrador único durante largas secciones de la novela. Dichas notas, a pie, metafóricamente, se convierten pues en un "exilio" de parte de la narración (al ser expresadas "fuera" del texto principal, dominado por la voz autorial que es, en cierto modo, "dictatorial"). Y es curioso cómo en la historia narrada, literalmente, a pie de página, se incide en ocasiones en figuras de resistencia al Trujillato: así, el escritor Juan Bosch, nombrado en la nota a pie número 30 (Díaz 2007: 250), Galíndez, en las notas a pie números 11 (Díaz 2007: 96) y 28 (Díaz 2007: 225), o las hermanas Mirabal, en la nota a pie número 7 (Díaz 2007: 83). Las notas a pie se convierten así, también por su contenido, en foco de resistencia al texto principal.

La intención de Díaz al utilizar, además, un largo número de referencias a la literatura de ciencia ficción, cómics, fantasía, etc., va más allá de una educación llamémosla "sentimental" como adolescente norteamericano: se trata, también, de la intención consciente de mostrar la experiencia de exilio, de alienación, a un amplio público lector no familiarizado con los modos narrativos de estos géneros populares, describiendo el horror, la locura y lo incomprensible del régimen y sus consecuencias sobre los De León desde la perspectiva desfamiliarizadora de géneros como la ciencia ficción. "What's more sci-fi than the Santo Domingo, what more fantasy than the Antilles?" (Díaz 2007: 6), se pregunta Oscar en la novela. El rechazo, al menos parcial, del canon sirve para liberar a estos autores de la diáspora, como reflexiona Díaz:

If you're a person writing about a Dominican diasporic experience, to hew too closely to canonical ideal of what literature is would limit you. The conventions of what is canonically known as literature can't hope to encompass these radical experiences that you undergo when living in a diaspora like the Dominican one. And sometimes the only way to describe these lived moments -the surreality and irreality of some of the things that people like myself have experienced- is through lenses like science fiction. (Lewis 2002) 


\section{Oscar es definido en el libro como alguien cuyo}

commitment to the Genres had become absolute [...]. Could write in Elvish [...] knew more about the Marvel Universe than Stan Lee, and was a role-playing game fanatic [...]. Dude wore his nerdiness like a Jedi wore his light saber [...]. Couldn't have passed for Normal if he'd wanted to. (Díaz 2007: 21)

El narrador, Yunior, intrigado por la obsesión del protagonista por la ciencia ficción, se pregunta si el amor de Oscar por este género no partirá, precisamente, de su experiencia diaspórica:

[I]t might have been a consequence of being Antillean (who more sci-fi tan us?) or of living in the DR [Dominican Republic] for the first couple of years of his life and then abruptly wrenchingly relocating to New Jersey -a single green card shifting not only worlds (from Third to First) but centuries (from almost no TV or electricity to plenty of both). After a transition like that I'm guessing only the most extreme scenarios could have satisfied. (Díaz 2007: 21-22)

Los cómics, además, tal y como expresa el crítico Albert Jordy Raboteau reflexionando sobre el texto de Díaz, "fed my imagination with larger than life characters of good and evil, with dramatic and cosmic battles between light and darkness, life and death, and especially power used for good vs. power used for domination" (Raboteau 2008: 920-921), algo más que relacionado con los temas que se tratan en la novela. En la narración, se colocan casi al mismo nivel de realidad (o irrealidad) las historias de superhéroes y supervillanos y la lucha y represión histórica que supone el régimen de Trujillo: se desestabiliza, así, la pretensión de realidad del discurso histórico sobre los episodios de represión política, y se pone al descubierto la incapacidad por parte de disciplinas manipulables (como ya sabemos que es la Historia) de captar una verdad objetiva, quedando dicha disciplina al nivel de las historias, en ocasiones profundamente maniqueas, de los cómics de superhéroes. Una reflexión, por más que apresurada, sobre los temas fundamentales de la literatura fantástica y de los cómics destacaría, precisamente, el exilio, relacionado con la búsqueda, como el tema fundamental de un gran número de obras de ambos géneros. Y es imposible no leer la vida de Oscar Wao como una novela de búsqueda, casi en términos jungianos, en la que el exilio interior al que le relegan sus dificultades para relacionarse con el mundo "normal" refleja el exilio (exterior, real) de su familia por la imposibilidad de seguir viviendo en Santo Domingo durante la época de la dictadura de Trujillo. Oscar es, durante su infancia, la promesa, para su entorno, de lo que ha de ser un joven dominicano, pero se "exilia" de ese contexto familiar y étnico al entrar en la adolescencia:

[I]n those blessed days of his youth, Oscar was something of a Casanova. One of those preschool loverboys who was always trying to kiss the girls, always coming up behind them during a merengue and giving them the pelvic pump, the first nigger to learn the perrito and the one who danced it any chance he got. Because in those days he was (still) a "normal" Dominican boy raised in a "typical" Dominican family, his nascent pimp-liness was encouraged by blood and friends alike 
[...]. In the DR [Dominican Republic] during summer visits to his family digs in Baní he was the worst, would stand in front of Nena Inca's house and call out to passing women -Tú eres guapa! Tú eres guapa! [...] It truly was a Golden Age for Oscar [but] [e] arly adolescence hit him especially hard [...] making him self-conscious, and his interest -in Genres!- which nobody had said boo about before, suddently became synonymous with being a loser with a capital L. Couldn't make friends for the life of him, too dorky, too shy, and [...] too weird (had a habit of using big words he had memorized the day before) [...]. He forgot the perrito, forgot the pride he felt when the women in the family had called him hombre. (Díaz 2007: 11-17, passim)

La metáfora en torno a la que se articula la obra desde el primer momento es el concepto de "Fukú", una maldición similar al mal de ojo que parece hundirse en el pasado animista de la isla, y que para el narrador ha perseguido al Caribe (y, específicamente, a los De León) desde la época de la colonización de La Española. El narrador habla de un "Fukú americanus", que se ha extendido desde la isla a todo el continente:

The arrival of Europeans on Hispaniola unleashed the fukú on the world, and we've all been in the shit ever since. Santo Domingo might be fukú's Kilometer Zero, its port of entry, but we are all of us its children, whether we know it or not.

(Díaz 2007: 1-2)

Existe a continuación en la obra una identificación explícita entre el fukú y la represión del régimen de Trujillo:

No one knows whether Trujillo was the Curse's servant or its master, its agent or its principal, but it was clear he and it had an understanding, that them two was tight. It was believed, even in educated circles, that anyone who plotted against Trujillo would incur a fukú most powerful, down to the seventh generation and beyond. If you even thought a bad thing about Trujillo, fuá, a hurricane would sweep your family out to sea, fuá, a boulder would fall out of a clear sky and squash you, fuá, the shrimp you ate today was the cramp that killed you tomorrow. (Díaz 2007: 3)

De forma específica, se conecta el fukú con la diáspora, convirtiendo el exilio en maldición y castigo: "My paternal abuelo believes that diáspora was Trujillo's payback to the pueblo that betrayed him. Fukú" (Díaz 2007: 5). El exilio dominicano formulado por Díaz recoge, así, los ecos del exilio mítico original del Jardín del Edén, que para Rossbach convierte para siempre el exilio en un castigo "inflicted [...] as a consequence of their [Adam and Eve's] action" (Rossbach 2008: 76). Al identificar la expulsión del paraíso a la experiencia del exilio, podemos derivar que el exiliado, aun habiendo escogido el exilio como opción legítima (en el sentido de que, pese a que suponga una renuncia moral a sus ideales políticos, permite seguir vivo), arrastre a menudo sensación de culpa por no haber aceptado, cediendo y dejando a un lado sus ideales, algo que le hubiera permitido seguir viviendo en el país: esto explica la visión idealizada, paradisiaca e idílica, que en ocasiones tiene el exiliado del país abandonado. 
Raboteau habla del silencio que rodea a las historias de exilio que se viven en la novela como "a legacy of silence required to survive under the constant threat of a brutal dictator $-\mathrm{a}$ Sauron ruling a nation as his own private Mordor" (Raboteau 2008: 921). Para Díaz, uno de los elementos que definen el libro es, precisamente, las cosas que faltan por decir, las que no se narran:

[T]he mother's entire childhood is missing [...]. The grandfather is disappeared by the government, imprisoned, tortured, a whole slice of his life disappeared [...]. The protagonist, Oscar, is always filtered thought this other narrator, Yunior [...] how do you put a story together from fragments and how you put a story together from absences [...]. The consequences of being in the Dominican Republic during the Trujillo Regime were unspeakable. (Roberts 2007)

El narrador principal de la obra, Yunior, sugiere que la única solución al poderoso hechizo del Fukú es un contra-hechizo, el "Zafa",

[The] only one way to prevent disaster from coiling around you, only one surefire counterspell that would keep you and your family safe. Not surprisingly, it was a word. A simple word (followed usually by a vigorous crossing of index fingers).

(Díaz 2007: 7)

Yunior plantea desde el principio de su narración que quizá "now as I write these words I wonder if this book ain't a zafa of sorts. My very own counterspell" (Díaz 2007: 7). Para Kalí Tal, gran parte de la literatura que hace referencia a traumas históricos (pero también familiares o individuales) como el exilio político está escrita "from the need to tell and retell the story of the traumatic experience, to make it 'real' both to the victim and to the community. Such writing serves both as validation and cathartic vehicle for the traumatized author" (Tal 1996: 21-22), al tiempo que identifica "displacement [as] the goal of any story, in degree; all fiction aims to usurp the real world with a world that is imagined" (Tal 1996: 21-22). El desplazamiento, la desubicación (genérica, intertextual, individual, social) es, pues, el tema fundamental en esta novela de Díaz, una obra sobre exilios, exteriores e interiores, textuales e intertextuales, sobre el trauma y lo incontable, sobre maldiciones que producen diásporas y desplazamientos, y en última instancia sobre el posible poder curativo (o, siguiendo a Yunior, el "Zafa") que pueden tener la palabra y la literatura.

\section{BIBLIOGRAFÍA}

ÁLVAREZ BORLAND, Isabel (2003): "Las raíces al desnudo: narradores cubanos en los Estados Unidos", en Laura P. Alonso Gallo y Fabio Murrieta (eds.), Guayaba Sweet: Literatura cubana en los Estados Unidos, pp. 37-52. Valencia: Aduana Vieja.

DÍAZ, Junot (2007): The Brief Wondrous Life of Oscar Wao. Nueva York: Riverhead Books.

- (2008): "Junot Díaz, the Devil and Me. Interview with Junot Díaz". Christchurch City Libraries. En: http://christchurchcitylibraries.com/Literature/People/D/DiazJunot/ [Consulta: $18 / 12 / 2010]$. 
FEHERVARY, Helen (2008): "Tales of Migration from Central America and Central Europe", en Marcus Bullock y Peter Y. Paik (eds), Aftermaths: Exile, Migration and Diaspora Reconsidered, pp. 15-32. New Brunswick, New Jersey: Rutgers.

GROSSMAN, Lev (2007): "What to Watch For: The Brief Wondrous Life of Oscar Wao" [on line]. Time Magazine. En: http://www.time.com/time/specials/2007/article/0,28804, 1655968_1655989_1656010,00.html [Consulta: 18/12/2010].

INK, Lynn Chun (2004): "Remaking Identity, Unmaking Nation: Historical Recovery and the Reconstruction of Community in In the Time of the Butterflies and The Farming of Bones". Callaloo, 27.3, pp. 788-807.

KAKUTANI, Michiko (2007): "Travails of an Outcast" [on line]. The New York Times, September 4, 2007. En: http://www.nytimes.com/2007/09/04/books/04diaz.html?_r= $1 \&$ scp $=3 \&$ sq $=$ junot + diaz + kakutani\&st $=$ nyt [Consulta: $10 / 12 / 2010]$.

LEWIS, Marina (2002): "Interview with Junot Diaz" [on line]. Other Voices, 36. En: http://webdelsol.com/Other_Voices/ [Consulta: 10/01/2011].

LIFSHEY, Adam (2008): "Indeterminacy and the Subversive in Representations of the Trujillato". Hispanic Review, 76.4, pp. 435-457.

O'BRIEN, George (1988): “The Muse of Exile: Estrangement and Renewal in Modern Irish Literature”, en María-Inés Lagos-Pope (ed.), Exile in Literature, pp. 82-101. Lewisburg: Brucknell University Press.

PÉREZ-FIRMAT, Gustavo (1994): Life on the Hyphen: The Cuban American Way. Austin: University of Texas Press.

RABOTEAU, Albert Jordy (2008): “Conversation with Junot Díaz (To the Woman in the Mountain Cabin)". Callaloo, 31, pp. 919-922.

ROBERTS, Caroline (2007): "Bostonist Interview: Junot Díaz, Author" [on line]. Bostonist. En: http://bostonist.com/2007/09/10/bostonist_inter_1.php [Consulta: 18/12/2010].

ROSSBACH, Stefan (2008): "On the Metaphysics of Exile", en Marcus Bullock y Peter Y. Paik (eds), Aftermaths: Exile, Migration and Diaspora Reconsidered, pp. 223-242. New Brunswick, New Jersey: Rutgers.

SHORTER, Daniel (2005): "Dominican Republic Ancestry Maps". Epodunk, the Power of Place. En: http://www.epodunk.com/ancestry/Dominican-Republic.html [Consulta: 04/01/2011].

SUAREZ, Lucía M. (2004): "Julia Alvarez and the Anxiety of Latina Representation”. Meridians: Feminism, Race, Transnationalism, 5.1, pp. 117-145.

TAL, Kalí (1996): Worlds of Hurt: Reading the Literatures of Trauma. Cambridge, MA: Cambridge University Press.

YING, Hao (2010): "Writing Wrongs" [on line]. Global Times. En: http://www.globaltimes.cn/www/english/metro-beijing/people/profile/2010-04/522054.html [Consulta: $18 / 12 / 2010]$. 